\title{
Multiscale approach to description of deformation and fracture of brittle media with hierarchical porous structure on the basis of movable cellular automaton method
}

\author{
Ig. S. Konovalenko \\ Institute of Strength Physics and Materials Science SB RAS, 2/4 Akademicheskii pr., Tomsk, 634021, Russia \\ igkon@ispms.tsc.ru
}

\author{
A. Yu. Smolin, S. G. Psakhie \\ Institute of Strength Physics and Materials Science SB RAS, 2/4 Akademicheskii pr., Tomsk, 634021, Russia \\ Tomsk State University, 36 Lenin pr., Tomsk, 634050, Russia
}

\begin{abstract}
An approach to multiscale description of deformation and fracture of brittle porous materials on the basis of movable cellular automaton method was proposed. The material characterized by pore size distribution function having two maxima was considered. The core of the proposed approach consists in finding the automaton effective response function by means of direct numerical simulation of representative volume of the porous material. A hierarchical two-scale model of mechanical behavior of ceramics under compression and shear loading was developed. Zirconia based ceramics with pore size greater than the average grain size was considered. At the first scale of the model only small pores (corresponding to the first maximum of the pore size distribution function) were taking into account explicitly (by removing automata from the initial structure). The representative volume and effective elastic properties of the porous material at this scale were evaluated. At the second scale of the model, big pores were taking into account explicitly, the parameters of the matrix corresponded to the ones determined at the first scale. Simulation results showed that the proposed multiscale model allows qualitatively and quantitatively correct describing of deformation and fracture of brittle material with hierarchical porous structure.
\end{abstract}

KEYwORDS. Deformation and fracture; Brittle materials; Porous ceramics; 2D modeling; Particle-based approach, Movable cellular automaton method.

\section{INTRODUCTION}

I $\mathrm{t}$ is well known that real porous materials are characterized by hierarchical pore structure and complex mechanical behavior, including fracture [1-3]. To study and describe these materials the information about the relationship of their structure and mechanical properties at various scales is needed. One of the ways of getting this information is numerical simulation. In the framework of one-scale approach direct taking into account all peculiarities of structure and mechanical behavior of a material at each scale seems to be impossible. Therefore the goal of this paper is development of the multiscale approach, represented in [4], and construction of the corresponding hierarchical model for describing deformation and fracture of nanostructured porous ceramics under compression and shear on the basis of movable cellular automaton method (MCA). Detailed description of the MCA method can be found in paper [5] published in this issue of the journal. Note, that this method have been chosen because it has successfully proved itself in studying 
mechanical behavior of brittle porous materials from initiation of the first damages till cracks propagation and complete failure [6-9]. The calculations were carried out for a model material having mechanical properties of nanocrystalline $\mathrm{ZrO}_{2}\left(\mathrm{Y}_{2} \mathrm{O}_{3}\right)$ (yttria-stabilized zirconia) with the average pore size greater than the average grain size and two maxima in its pore size distribution function $[2,3]$.

The hierarchical model of the material with properties of the ceramics under consideration was constructed in several stages. At the first stage, the response of the ceramic material at the microscale $(20 \div 250 \mu \mathrm{m})$ was simulated with explicit taking into account the porous structure of the material under various types of mechanical loading and the representative volume of this scale was determined. The result of the first stage was determination of the response function parameters of macroscale movable automata, corresponding to representative volume of the microscale. At the second stage, similar calculations were carried out at the macroscale with explicit taking into account of the porous structure of this scale. Data on the porous structure of lower scale were allowed for in the effective automata response functions defined at the first stage. At the final third stage, qualitative and quantitative verification of the developed model at the macroscale was made including comparison of simulation and experimental data.

\section{DETERMINING THE REPRESENTATIVE VOLUME OF THE MICROSCALE AND THE MACROSCALE EFFECTIVE RESPONSE FUNCTION}

A t the microscale of the proposed model, the representative volume is determined by analyzing the convergence of elastic and strength characteristics of porous model specimens with an increase in their dimension. Modeling of six groups of porous ceramics specimens under uniaxial compression and simple shear was performed. All the specimens in each group had the same dimension, but different pore distribution in space. Each group was consisted of six plane square specimens. Specimens under consideration had dimension (square side) of 20, 60, 100, 150, 200 and $250 \mu \mathrm{m}$ according to the groups. It was supposed that all pores in the ceramics under investigation, as well as the model material, were equiaxed. The pore size of the model material, according to the maximum of the ceramics pore size distribution function, was equal to $3 \mu \mathrm{m}[2,3]$. Diameter of the movable cellular automata, according to the average grain size, was equal to $1 \mu \mathrm{m}[2,3]$. The pore structure of the specimens was specified by randomly removing individual automata and their six nearest neighbors. The total porosity for all the specimens was equal to 7\% [2, 3]. Typical initial structure of one of the model specimens is shown in Fig. 1.

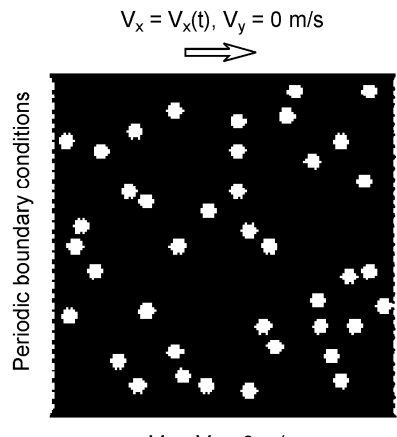

$V_{x}=V_{y}=0 \mathrm{~m} / \mathrm{s}$

(a)

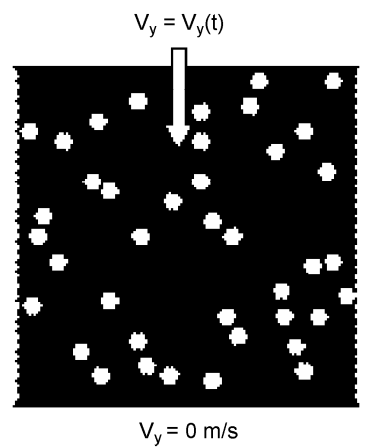

(b)

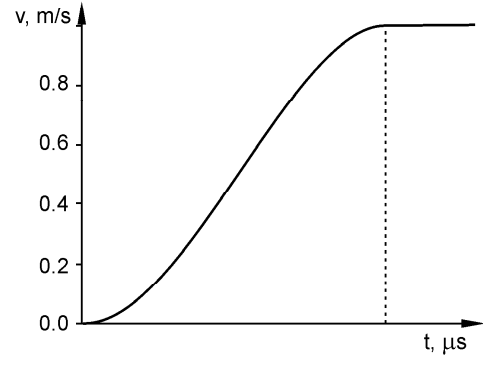

(c)

Figure 1: Initial structure of a model specimen with a size of $\mathrm{h}=60 \mu \mathrm{m}$ and scheme of loading for shear (a) and uniaxial compression (b); the plot of velocity vs time for the upper automaton layer of the specimen (c).

The shear loading was simulated by setting up one and the same horizontal velocity to all automata in the upper layer with automata of the lower layer being rigidly fixed (Fig. 1,a,c). At the initial stage, the velocity of automata of the upper layer was increased by the sinusoidal law from 0 to $1 \mathrm{~m} / \mathrm{s}$ and then was assumed to be constant. This scheme ensured a quasisteady regime of loading and allowed dynamic effects to be eliminated until the first damage appeared. Duration of the loading velocity increase depended on the size of the specimen and was determined by preliminary calculations. All the samples had periodic boundary conditions in the direction of shear loading. The uniaxial compression loading was simulated by setting up one and the same velocity in vertical direction (up to $1 \mathrm{~m} / \mathrm{s}$ ) to all automata in the upper layer (Fig. 1,b,c). The vertical velocity of automata in the lower layer were set to zero. Displacements in horizontal direction were allowed for automata in the both lower and upper layers. The lateral surfaces of the specimen were free. The problem was solved under plane strain conditions. The response function of automata corresponded to the loading 
diagram for nanocrystalline $\mathrm{ZrO}_{2}\left(\mathrm{Y}_{2} \mathrm{O}_{3}\right)$ with total porosity of $2 \%$ and an average pore size to be equal about the grain size $[2,3]$. Shear modulus $G$ and Poisson ratio $\nu$ of the movable cellular automaton were equal to $59.2 \mathrm{GPa}$ and 0.3 correspondingly. Inter-automaton bond rupture criterion used in calculations was formulated as a threshold value for intensity of shear stresses [10].

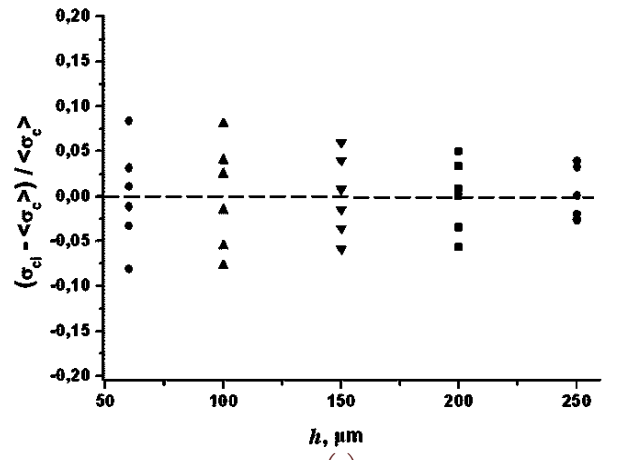

(a)

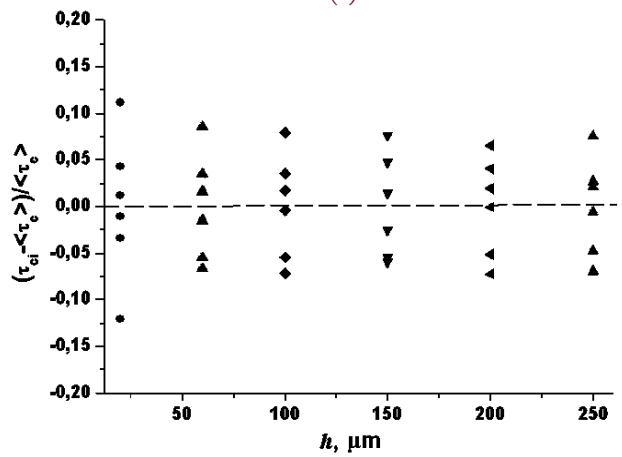

(c)

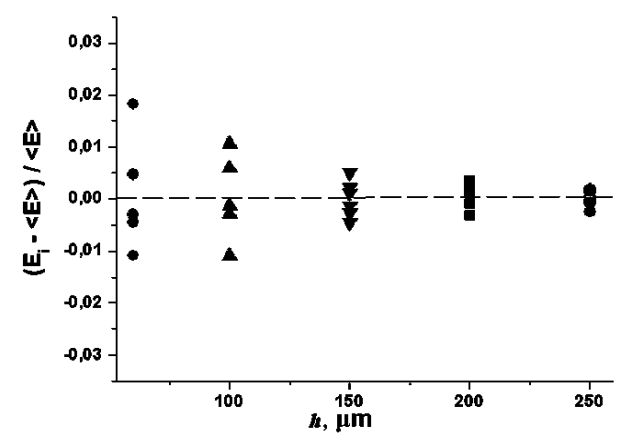

(b)

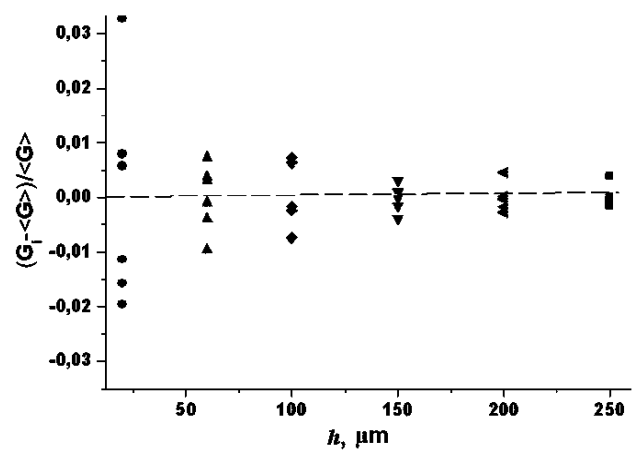

(d)

Figure 2: Relative deviation of compression modulus $E_{\text {eff, }}$ shear modulus $G_{\text {eff, }}$ compression strength $\sigma_{\mathrm{c}}$ and shear strength $\tau_{\mathrm{c}}$ of the model specimens from the corresponding mean values $\left\langle E_{\text {eff }}\right\rangle,\left\langle G_{\text {eff }}\right\rangle,\left\langle\sigma_{c}\right\rangle,\left\langle\tau_{c}\right\rangle$ under uniaxial compression (a, b) and shear (c, d).

Convergence analysis of mechanical properties for the porous model specimens with increase of their size was performed in terms of estimated deviation of effective elastic and strength properties of the specimens (modulus of compression $E_{\text {eff, }}$ shear modulus $G_{\text {eff }}$, compression strength $\sigma_{\mathrm{c}}$ and shear strength $\tau_{\mathrm{c}}$ obtained from simulated loading diagram) from the corresponding mean values in the groups $\left\langle E_{\text {eff }}\right\rangle,\left\langle G_{\text {eff }}\right\rangle,\left\langle\sigma_{\mathrm{c}}\right\rangle$ and $\left\langle\tau_{\mathrm{c}}\right\rangle$. The specimen size for which the deviation did not exceed $3 \%$ for $E_{\text {eff }}, G_{\text {eff }}$ and $15 \%$ for $\sigma_{\mathrm{c}}, \tau_{\mathrm{c}}$ was accepted as the size of representative volume. The results of simulation (Fig. 2) showed nonlinear convergence of strength and elastic properties of the model specimens. For the model specimens with the side of $150 \mu \mathrm{m}$ relative deviations of $E_{\text {eff }}, G_{\text {eff }}, \sigma_{\mathrm{c}}$ and $\tau_{\mathrm{c}}$ from the corresponding group average values were 0.51 , 0.38, 6.0 and 7.6\% correspondingly. These values did not exceed the prescribed limits. Thus, it proved that the porous specimens with dimension of $150 \mu \mathrm{m}$ are representative volumes of the model material under consideration. The values of $\left\langle E_{\text {eff }}\right\rangle$ and $\left\langle\sigma_{\mathrm{c}}\right\rangle$ were taken as the parameters of response function of automata at macroscopic scale. Slight deviations of these values $(-9.5 \%$ and $36.0 \%$ ) from ones evaluated experimentally are related with two-dimensional formulation of the problem and incomplete correspondence of the model pore morphology with the one of real ceramics. Therefore the corresponding characteristics of response function were corrected by means of the correction ratio. The conversion from the elastic modulus determined from the loading diagram under plane strain conditions EPSS to the Young's modulus was based on the ratio $E=E_{P S S}\left(1-v^{2}\right)[11]$.

\section{SIMULATIONS AT MACROSCALE}

$\mathrm{t}$ macroscale the calculations were carried out for nine square porous specimens with the dimension of $22.5 \mathrm{~mm}$. According to the representative volume determined at the previous stage the diameter of movable cellular automata at this scale was equal to $150 \mu \mathrm{m}$. The information about structure and strength properties of the 
material was transferred from micro- to macroscale by means of the response function with the parameter values corresponding to the loading diagrams of the representative volume of the material at microscale. The response function of automata was chosen to be a linear one and characterized by two parameters: the maximal value of specific resistance force (corresponded to strength limit) and the elastic parameter (corresponded to Young's modulus). The values of these parameters determined at the first stage were found to be equal to $846 \mathrm{MPa}$ and $112 \mathrm{GPa}$ correspondingly. Explicit setting up of pore structure of the material at macroscale scale, loading conditions and assumption about stress state were similar with that of the first stage. According to the pore size distribution function the explicit porosity of the macroscale specimens was equal to $28 \%$ and the pore size was equal to $450 \mu \mathrm{m}[2,3]$.

\section{VERIFICATION OF THE MODEL}

工

et us assume the model to be successfully verified (i.e. the model represents the main features of the ceramics under investigation) if the simulation results satisfy the following criteria: 1) the loading diagram of the modeled specimen is linear in elastic region and contains horizontal section corresponding to quasi-ductile fracture for porosity greater than $20 \%$;2) qualitative correspondence of the fracture patterns of the modeled specimens to real ceramics; 3) strength properties of the modeled specimens belong to a certain value interval found from experimental data.

The loading diagrams which are typical for all the model specimens in case of different types of mechanical loading are presented in Fig. 3. On these diagrams one can see several parts. The first linear part, corresponding to elastic deformation of the specimen, is typical for brittle materials with any value of porosity. The next part is still ascending but insignificantly, it also contains multiple stress "oscillations" (only under uniaxial compression, Fig. 3, a). This part corresponds to repetitive processes of damage generation, local cracking and subsequent elastic deformation of the material all over the entire specimen. The last part of the diagram is descending. It corresponds to macrocrack propagation as well as generation of separate multiple damages. In shear loading (Fig. 3, b) one can see another ascending sections of the curve with breakdowns and subsequent drop-down on the plotted diagram after the above mentioned parts. Under constrained deformation conditions, it is to these portions of the diagram that the development of a system of macrocracks in the specimen corresponds, and the first descending portion corresponds to nonrecurring generation of damages throughout the entire specimen and their development without the formation of a system of macrocracks.

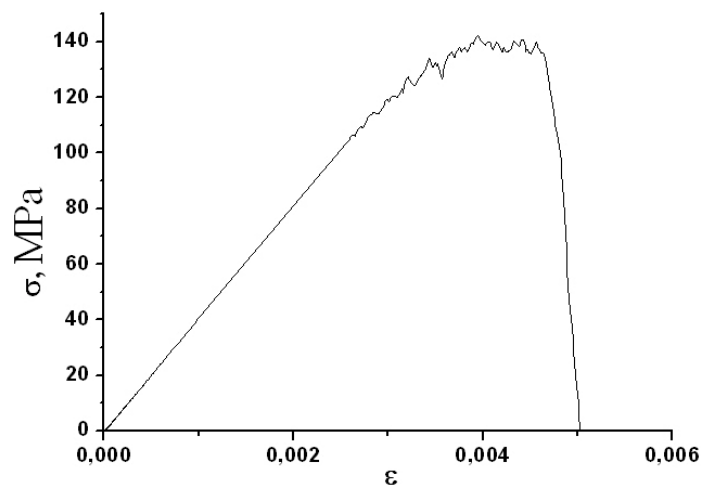

(a)

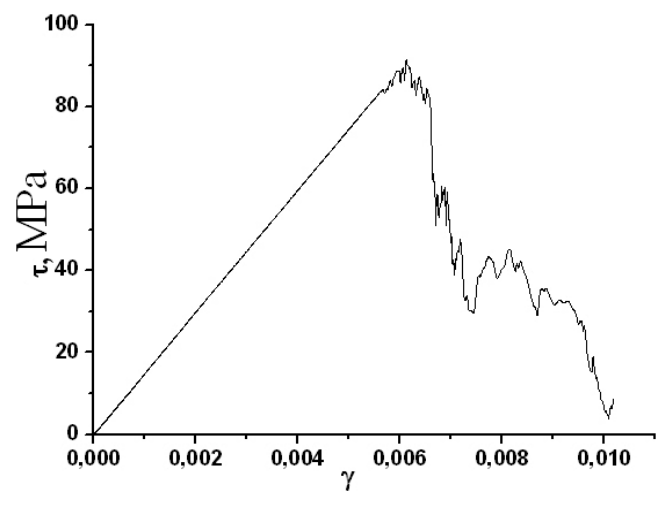

(b)

Figure 3: Loading diagrams of the model specimen with dimension of $22.5 \mathrm{~mm}$ under uniaxial compression (a) and shear loading (b).

It should be noted, that the horizontal plateau on the compression diagram of the brittle specimens (Fig. 3, a) reveals their quasi-ductile fracture, which occurs only when porosity of the specimen is greater than $20 \%$ [9]. The extent to which these properties show up is proportional to the length of the given portion (plateau) and is different for different model specimens. A decrease in the length of this diagram portion points to the proximity of fracture to brittle fracture. It would appear reasonable that both the transition to quasi-ductile fracture and the extent to which it develops are determined by certain critical local porosity of the specimens. The critical local porosity is associated, in particular, with the total specimen porosity and with the pore shape and size. It is worthy of note that quasi-ductile fracture in this case is completely determined by the geometric factor, because the model takes into account neither phase transitions, nor rearrangement of the material lattice. 
Typical loading diagrams of brittle porous solids under shear and uniaxial compression are presented in Fig. 4 [2, 9]. Comparison of the diagrams in Fig. 3 with corresponding diagrams of brittle porous solids in shear and uniaxial compression [2,9] shows their good qualitative agreement. Thus, the first macroscale criterion of model verification, which is in the loading diagram correlation, is fulfilled.

Typical fracture pattern of the model specimens, represented as inter-automaton bond net at the time of first macrocrack propagation, are shown in Fig. 5. Under uniaxial compression the specimen failure occurred due to generating therein the asymmetrical system of macrocracks with complex propagation path. In addition, generation of multiple separated fracture regions took place near the macrocracks.

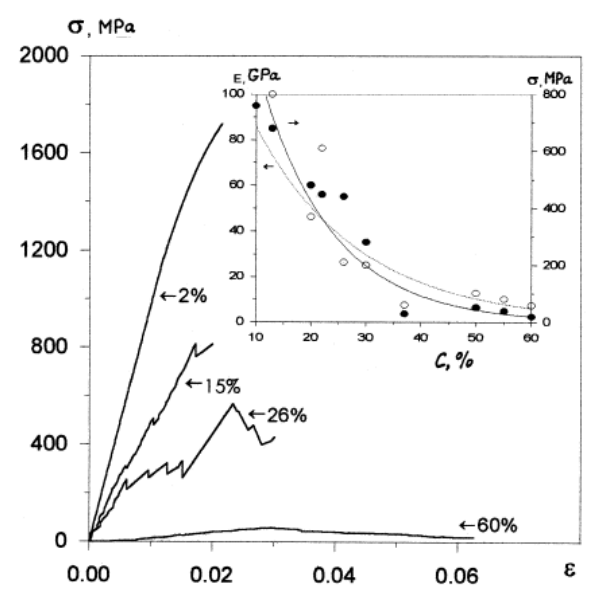

(a)

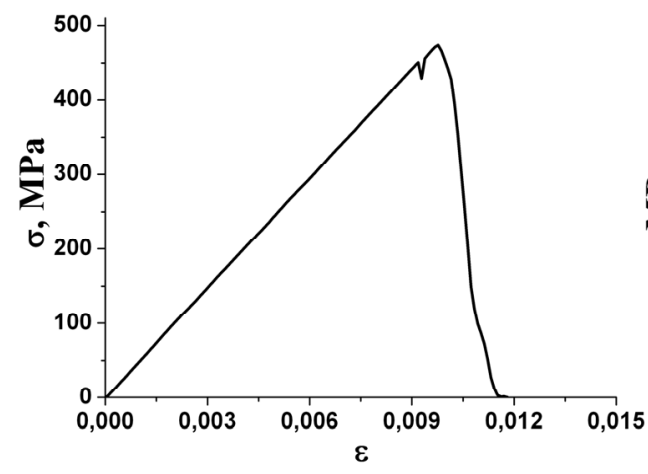

(b)

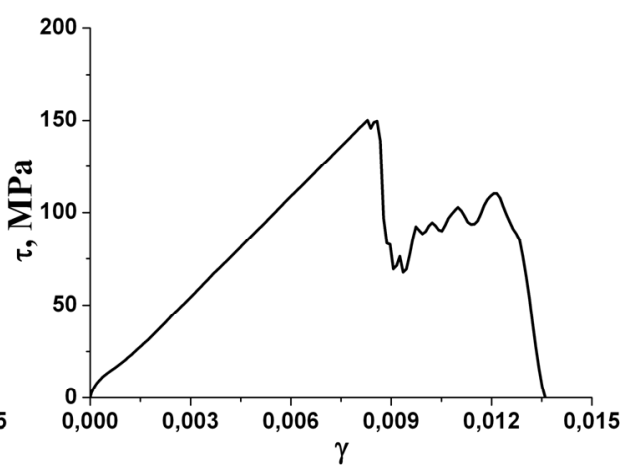

(c)

Figure 4: Experimental loading diagrams of $\mathrm{ZrO}_{2}$ ceramic specimen with different value of porosity under uniaxial compression (a) [2] and corresponding diagrams obtained from numerical simulation (b and c) [9].

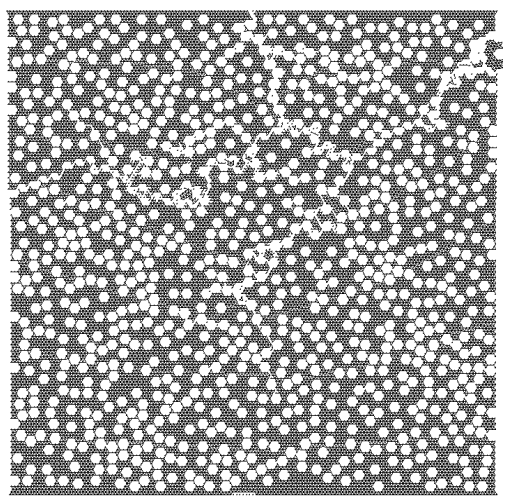

(a)

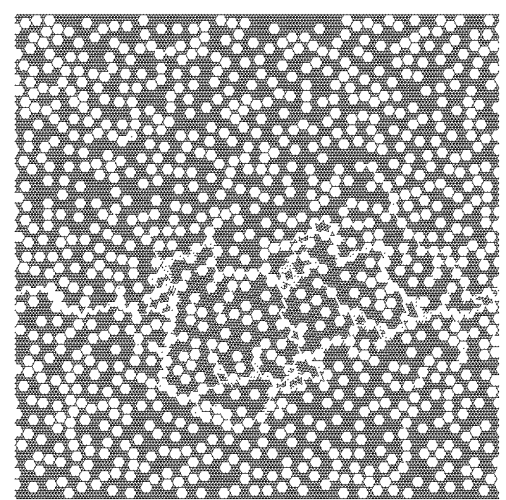

(b)

Figure 5: Fracture patterns of the model specimen with the porosity of $28 \%$ at the moment of the first macrocrack propagation under uniaxial compression (a) and shear loading (b). 
In the case of quasi-ductile fracture (Fig. 3, a) damage generation and crack growth occurred locally, in several regions of the sample, characterized by the highest value of local porosity (and the least thickness of web between isolated pores). Until a certain moment, some cracks were not merged into main crack, the stage of its growth were somehow elongated. It led to extensive local cracking of the material without losing the integrity of the sample and, consequently, to a substantial dissipation of elastic energy and decreasing of the effective elastic properties of the material (of the whole specimen). Thus, the second criterion of the constructed model verification is fulfilled.

To verify the third criterion of the model verification the average value of the effective elastic modulus $\left.\left(<\mathrm{E}_{\text {eff }}\right\rangle\right)$ and maximum specific resistance force to loading under uniaxial compression were calculated. Then they were compared with the corresponding values found from real experiments. It was shown that the deviation of $\left\langle\sigma_{s_{-} \text {eff }}\right\rangle$ and $\left\langle\mathrm{E}_{\text {eff }}\right\rangle$ for the model samples from the experimental data did not exceed $30 \%$ and $12 \%$, respectively. It is a rather good accuracy for simulating highly porous media in plane approximation. This indicates to a good quantitative agreement of the calculations and the experiment and means that the third criterion of the model verification is fulfilled.

Thus, a two-scale model of porous ceramics with bimodal pore size distribution function was constructed herein based on a multiscale approach to numerical simulation and validated against available experimental data.

\section{CONCLUSIONS}

A multiscale approach to numerical simulation of porous materials is developed on the basis of movable cellular automaton method. The hierarchical two-scale model constructed using the proposed approach can adequately describe deformation and fracture of the porous zirconia ceramics under mechanical loading. Since the proposed approach is sufficiently general, then, if it is necessary, a heterogeneous material containing more than two structural scales can be also simulated on the basis of this approach.

\section{ACKNOWLEDGEMENTS}

his study was supported by the Russian Foundation for Basic Research, project No. 12-08-00379-a.

\section{REFERENCES}

[1] Global Roadmap for Ceramics: Proceedings of 2nd International congress on ceramics (ICC2). Alida Belosi and Gian Nicola Babini (Eds.). Institute of Science and Technology for Ceramics, National Research Council, Verona (Italy), (2008).

[2] S.P. Buyakova, Han Wei, Li Dunmy et al., Tech. Phys. Lett., 25(9) (1999) 695.

[3] S.N. Kulkov, S.P. Buyakova, V.I. Maslovskii, Vestnik TGU, 13 (2003) 34 [in Russian]

[4] Ig.S. Konovalenko, A.Yu. Smolin, S.G. Psakhie, Phys. Mesomech., 13(1-2) (2010) 47.

[5] S. Psakhie, E. Shilko, A. Smolin, S. Astafurov, V. Ovcharenko, Frattura ed Integrità Strutturale, 24 (2013) 26.

[6] Ig.S. Konovalenko, A.Yu. Smolin, In: Proc. of the XXXVI Summer School "Advanced problems in mechanics (APM' 2008)" D. A. Indeitsev, A. M. Krivtsov (Eds.), Institute for problems in mechanical engineering, St. Petersburg, (2008) 352.

[7] A.Yu. Smolin, Ig.S. Konovalenko, S.N. Kul'kov, S.G. Psakhie, Tech. Phys. Lett., 32(9) (2006) 738.

[8] A.Yu. Smolin, Ig.S. Konovalenko, S.N. Kulkov, S.G. Psakhie, Izv. vuzov Fizika, 49(3) (2006) 70 [in Russian].

[9] Ig.S. Konovalenko, Theoretical Study of Deformation and Fracture of Porous Materials for Medicine and Biomechanical Structures, Cand. Degree Thesis (Phys\&Math), IFPM SO RAN, Tomsk, (2007) [in Russian].

[10] L.M. Kachanov, The Foundations of Fracture Mechanics, Nauka, Moscow, (1974) [in Russian]

[11] L.I. Sedov, A Course in Continuum Mechanics, Wolters-Noordhoff, Groningen, (1971). 